Revista de Psicología y Educación / Journal of Psychology and Education, 2019, 14(1), 1-15 (www.rpye.es) Doi: https://doi.org/10.23923/rpye2019.01.167

ISSN: $1699-9517$

\title{
¿Qué motivos tienen los estudiantes de Bachillerato para elegir una carrera universitaria?
}

Luis. J. Rodríguez-Muñiz, Debora Areces*, Javier Suárez-Álvarez, Marisol Cueli y José Muñiz

Universidad de Oviedo

Resumen: La elección de una carrera universitaria supone el resultado de un proceso de autodescubrimiento en el que intervienen diversas variables. El objetivo central de la presente investigación es analizar cuáles son los motivos que llevan a los estudiantes de Bachillerato a elegir unos determinados estudios. Para ello, se ha diseñado una escala que permite medir los motivos de elección de estudios universitarios. Participaron 2005 estudiantes (46.10\% hombres y 53.90\% mujeres) de segundo curso de Bachillerato con edades comprendidas entre los 17 y los 24 años $(M=17.56$; DT $=.77$ ) que suponen el $44.95 \%$ del total de los centros educativos asturianos que imparten diferentes ramas de Bachillerato: Ciencias y Tecnología (54.4\%), Ciencias Sociales (30\%), Humanidades (14.2\%) y Artes (1.4\%). La nueva escala presenta unas adecuadas propiedades psicométricas y ha permitido comprobar qué tipo de motivos tienen un mayor peso en la elección de estudios universitarios. Las motivaciones intrínsecas relacionadas con las preferencias personales de los estudiantes fueron las más influyentes. Sin embargo, motivaciones de tipo extrínseco como la tradición familiar o la facilidad de la carrera, fueron las que menos influyen en la elección de un determinado grado universitario.

Palabras clave: Grado Universitario, Motivos, Cuestionario, Bachillerato, Orientación Vocacional.

\begin{abstract}
What motives have high school students for choosing a college degree?
Abstract: Choosing a college degree is the result of a self discovery process in which many variables are involved. The main aim of the present research is to analyze what motives lead to high school students to choose a college career. To this end, a scale was designed in order to measure the motives of choosing a college degree. 2005 high school students (46.10\% males y 53.90\% females) aged 17 to 24 ( $M=$ 17.56 ; DT $=.77$ ) participated in the study and represent $44.95 \%$ from the total of school centers of the Principality of Asturias). These centers teach the post-compulsory education which is divided into different branches, depending on the focus of study: Science and Technology (54.4\%), Social Sciences (30\%), Humanities (14.2\%), and Arts (1.4\%). The new scale has adequate psychometric properties and it has allowed to check the main motives for choosing a college degree. The most influential intrinsic motivations are those related to personal preferences of students. On the other hand, the extrinsic motivations such as family tradition or the ease of the course were the least influential motivations when choosing a university degree course.
\end{abstract}

Keywords: University Degree, Motives, Questionnaire, High School Students, Vocational Guidance.

La elección de una carrera universitaria es un momento decisivo en la vida de todo estudiante y supone el resultado de un proceso de autodescubrimiento donde intervienen diferentes variables (Lorenzo, Argos, Hernández,

Recibido: 04/05/2018 - Aceptado: 09/07/2018 - Avance online: 25/07/2018 *Correspondencia: Debora Areces.

Universidad de Oviedo.

C.P: 33003, Oviedo, España.

E-mail: arecesdebora@uniovi.es

Rodríguez-Muñiz, L. J., Areces, D., Suárez-Álvarez, J., Cueli, M. y Muñiz, J. (2019). ¿̇Qué motivos tienen los estudiantes de Bachillerato para elegir una carrera universitaria? Journal of Psychology and Education, 14(1), 1-15, https://doi.org/10.23923/ pye2019.01.167 y Vera, 2014). Como señalan Pérez, Talavera y Ramos (2013), cuando un estudiante toma una buena decisión sobre su futuro académico se genera el proceso de confirmación de la misma en el que el estudiante se encuentra satisfecho y motivado. Por el contrario, la elección errónea de un determinado grado universitario, puede llevar a dos tipos de consecuencias. La primera y más negativa, sería el abandono de los estudios universitarios. Mientras que la segunda,

1699-9517/@ 2019 Asociación Científica de Psicología y Educación (ACIPE).

Publicado por Consejo General de Colegios Oficiales de Psicólogos, España. Este es un artículo Open Access bajo la CC BY-NC-ND licencia (http://creativecommons.ord/ licencias/by-nc-nd/4.0/) 
consistiría en volver a plantearse el estudio de otra carrera universitaria, lo que supondría un desfase temporal respecto a los compañeros que acertaron con su decisión. En este sentido, estudios como el de Canales y Rios (2018) señalan que la falta de claridad vocacional es uno de los factores más mencionados por los estudiantes a la hora de explicar la deserción temporal de los jóvenes universitarios. Por tanto, identificar los motivos que influyen en la elección de una carrera universitaria puede ser clave para prevenir la deserción educativa del alumnado universitario. Según datos estadísticos del Ministerio de Educación, esta realidad es una constante en todas las comunidades autónomas, donde en algunas de ellas el abandono de los estudios sin finalizar roza el 40\% de los estudiantes (Álvarez, Cabrera, González, y Bethencourt, 2006). Por este motivo, cabe pensar que la orientación educativa es un elemento clave e indispensable dentro del contexto de la educación, en el que los estudiantes deben disponer de información clara sobre los distintos estudios, y así tomar una buena decisión sobre su futuro universitario. Es por ello, que la orientación vocacional no solo debe consistir en una simple guía conductora, sino que su principal utilidad versa en que la persona se conozca a sí misma, identificando el mundo que le rodea, y actuando en calidad ciudadana responsable, motivada y productiva no solo en una sociedad laboral sino también en su contexto personal (Martínez et al., 2016).

$\mathrm{Si}$ bien han sido varios los estudios que han diseñado instrumentos para orientar a los estudiantes en la elección de grados universitarios, han sido pocos los instrumentos e investigaciones que han tenido como objetivo principal la búsqueda de variables, metas y motivaciones que influyen en los estudiantes en el momento de decidirse por una determinada carrera. La mayoría de estas investigaciones suelen analizar los motivos de estudiantes pertenecientes a unos estudios en concreto (Conroy, Elliot, y Pincus,2009; Deemer, Martens,y Buboltz, 2010; Lerchundi, Núñez del Río, y González-Tirados, 2015; Savage, Bich, y Noussi, 2011 ), lo que impide la formulación de conclusiones generales acerca de los factores que influyen en la decisión de los estudiantes universitarios en su conjunto. En términos generales, a pesar de que estas investigaciones han utilizado poblaciones de estudiantes diferentes, todas ellas coinciden en señalar la influencia de determinados tipos de motivaciones en la elección de estudios universitarios. Concretamente, en España se llevaron a cabo diferentes investigaciones en estudiantes procedentes de los grados de Psicología, Biología y Derecho (Candela, 2008; Gámez y Marrero, 2000, 2003; López, González,y Rodríguez, 2009;) donde se observó cómo estos habían tomado la decisión de acuerdo a cinco factores: afiliación e interés por las relaciones, logro y prestigio, poder e influencia, superación de problemas afectivos y motivación extrínseca; cuyo valor explicativo varió en función de la población analizada. Más específicamente, otros autores (De la Mella Quintero, Díaz, y Pérez, 2013; Soria, Guerra, Giménez, y Escanero, 2006) se han centrado exclusivamente en el grado de Medicina, quienes detectaron que los factores más influyentes en la elección de la carrera son: la influencia social, el reconocimiento familiar y la vocación.

En esta línea, existen otros estudios que han tratado de identificar los factores influyentes en la elección de estudios universitarios de forma general, teniendo como muestra alumnado de diferentes estudios universitarios. Skatova y Ferguson (2014) diseñaron y aplicaron un cuestionario denominado: "Motivations Influencing Course Choice: MICC" que recogía los cuatro tipos de motivaciones más influyentes en la elección de una carrera universitaria: motivación altruista (helping motivation), motivación de logro y prestigio (career motivation), motivación intrínseca (intrinsic motivation), motivación de poder (loafing motivation). Estos autores comprobaron que cada una de estas motivaciones tenía mayor o menor poder predictivo en la toma de decisiones según el tipo de grado elegido. Por ejemplo, los estudiantes que eligieron la carrera de medicina respondían principalmente a motivaciones de tipo altruista e intrínseca, 
mientras que, por su parte, aquellos alumnos que eligieron una ingeniería solían mostrar una mayor predilección por motivaciones de poder y prestigio. Estos resultados concuerdan con otros estudios precedentes (Bowdeny Wood, 2011 ; Lubben, Davidowitz, Buffler, Allie, y Scott, 2010) que habían comprobado que tanto los rasgos personales como la influencia social son factores determinantes en la toma de decisiones sobre el futuro académico de los estudiantes.

Recientemente Peró, Soriano, Capilla, Olmos, y Hervás (2015) han llevado a cabo una investigación en ocho Universidades Españolas con el fin de comprender qué tipo de variables intervienen en la elección de una determinada carrera. Para ello, han aplicado un cuestionario diseñado ad hoc para el que se compone seis factores: (a) prestigio de la Universidad, (b) salidas laborales, (c) consideración social, (d) aspectos vocacionales, (e) influencia social y ( $f$ ) ubicación geográfica. Los resultados obtenidos reflejaron que los estudiantes pertenecientes a la rama de educación o salud daban más importancia a aspectos vocacionales, mientras que el alumnado de ingeniería o arquitectura valoraba más cuestiones relativas a las salidas laborales o la utilidad percibida de la carrera. La mayoría de estas investigaciones han utilizado muestras de alumnado universitario. Se trata, por tanto, de estudiantes que han informado retrospectivamente sobre los motivos de elección de carrera universitaria, lo que puede sesgar sus opiniones con el propósito de justificar las elecciones realizadas.

El objetivo central de este estudio consiste en comprobar qué motivos son más influyentes en la elección de una carrera universitaria desde una perspectiva prospectiva (futuros estudiantes universitarios), con objeto de obtener un conocimiento más profundo sobre las variables implicadas en el proceso de elección de estudios universitarios. Para ello, se diseñó y aplicó un nuevo instrumento de medida que ha permitido recoger el tipo de motivos que influyen en la elección de los estudios universitarios. El nuevo instrumento de medida fue desarrollado teniendo en cuenta las investigaciones previas, así como el análisis psicométrico en función de diferentes pilotajes a nivel cualitativo y cuantitativo. De este modo, es esperable que la nueva escala posea unas buenas propiedades psicométricas y permita recoger objetivamente los principales motivos que llevan a elegir una carrera u otra. En este sentido, de acuerdo a los resultados de investigaciones previas (Peró et al., 2015; Skatova y Ferguson, 2014), cabe esperar que los motivos con mayor influencia en la elección de estudios universitarios estén relacionados con las preferencias académicas y personales de los estudiantes. Conocer tales motivos permitirá disponer de información relevante para mejora los programas de orientación vocacional, cuya función principal de la orientación vocacional, pasa por capacitar a los estudiantes para que manejen con recursos eficientes, un mundo interno y profesional cada vez más complejo (Areces, Rodríguez-Muñiz, Suárez-Álvarez, De la Roca, y Cueli, 2016; Mendoza-León y Rodríguez, 2008)

\section{MÉTODO}

\section{PARTICIPANTES}

Participaron un total de 2005 estudiantes (46.10\% hombres y $53.90 \%$ mujeres) de segundo curso de Bachillerato con edades comprendidas entre los 17 y los 24 años $(M=$ 17.56; $D T=.77)$. Este alumnado procede de 49 centros educativos del Principado de Asturias (el 79\% de la muestra pertenece a un centro público, el $10.1 \%$ a un centro concertado y el $10.9 \%$ a un centro privado), donde cursan diferentes modalidades de Bachillerato: Ciencias y Tecnología (54.4\%), Ciencias Sociales (30\%), Humanidades (14.2\%) y Artes (1.4\%). De este modo, teniendo en cuenta que en Asturias hay un total de 109 centros que imparten Bachillerato, la muestra participante en esta investigación representa el $44.95 \%$ de la población asturiana, por lo que se trata de una muestra amplia en la participaron aquellos centros educativos que aceptaron a participar de forma voluntaria en el estudio. 


\section{INSTRUMENTO DE MEDIDA}

Los miembros del Servicio de Información y Orientación Universitarios (SIOU), que agrupa a los servicios de información y orientación de las Universidades Españolas y está constituida como una red de técnicos en el seno de la Conferencia de Rectores de las Universidades Españolas (CRUE-Universidades Españolas), coincidieron en señalar la necesidad de diseñar un instrumento que permitiera conocer los motivos más relevantes en el alumnado de Bachillerato sobre la elección de una determinada carrera universitaria. En concreto, el grupo de trabajo sobre guías y herramientas para la orientación universitaria de la Red SIOU detectó la necesidad de dotar a estos servicios de una herramienta no solo teórica sino de carácter eminentemente aplicado para llevar a cabo su trabajo diario, informando y orientando a los estudiantes de Bachillerato y universitarios. Hasta el momento, si bien se habían encontrado con escalas nacionales e internacionales que trataban de medir las motivaciones más influyentes en la elección de una carrera universitaria, éstas se aplicaban en los primeros cursos de grado, y eran adaptadas para cada grado de forma específica.

Por esta razón, se llevaron a cabo diferentes reuniones de expertos, con el objetivo de crear un nuevo instrumento que fuera administrado en un momento próximo a la toma de decisión (segundo de Bachillerato). El desarrollo de los ítems se realizó siguiendo las principales directrices y estándares internacionales para la construcción de test (American Educational Research Association, American Psychological Association y National Council on Measurement in Education, 2014; Haladyna y Rodríguez, 2013; Moreno, Martínez y Muñiz, 2015). En primer lugar, se redactó un largo listado de motivaciones de diferente tipología que podrían ser relevantes en el proceso de elección de estudios universitarios, estas motivaciones (tanto de tipo extrínseco como intrínseco) se formularon en base a la experiencia y opinión de los diferentes expertos, así como en base a motivaciones incluidas en estudios que han sido previamente mencionados (Bowdeny Wood, 2011 ; Lubben, Davidowitz, Buffler, Allie, y Scott, 2010; Skatova y Ferguson, 2014) los cuáles hacen referencia a cuatro grandes tipos de motivaciones: motivación altruista, motivación de logro y prestigio, motivación intrínseca y motivación de poder. Posteriormente, basándose en el listado obtenido, expertos en psicometría se encargaron de diseñar y ajustar el cuestionario para ser aplicado en un primer estudio piloto. De este modo, la primera versión del mismo fue administrada a dos centros educativos (uno público y otro privado) con el propósito de comprobar la comprensibilidad y claridad de los ítems.

Por último, a partir de los resultados del estudio piloto, se llevaron a cabo diferentes modificaciones que permitieron obtener la versión final del cuestionario conformado por ítems tipo likert. Esta versión final (Anexo 1) se compone dos partes claramente diferenciadas. La primera parte del cuestionario se compone de una serie de preguntas iniciales con el fin de recoger información sobre aspectos generales (como la edad, el género, o la modalidad de Bachillerato) así como de tipo personal y familiar (el nivel de estudios que poseen sus padres, las perspectivas futuras de ir a la Universidad y el grado universitario en el que quieren ingresar). Posteriormente, en la segunda parte, los estudiantes deben cumplimentar una escala formada por 16 ítems tipo Likert de 10 puntos, donde 1 significa estar totalmente en desacuerdo con el motivo de elección de estudios y 10 totalmente de acuerdo.

\section{PROCEDIMIENTO}

Para la aplicación de la escala, se contactó con los equipos directivos y departamentos de orientación de varios centros educativos del Principado de Asturias que imparten Bachillerato (a través del correo electrónico y llamadas telefónicas), quienes fueron informados de los objetivos y del procedimiento del estudio, así como de su carácter voluntario y anónimo. De esta forma, aquellos que aceptaron participar en el mismo, recibían un documento explicativo 
sobre cómo administrar el instrumento, y aquellos centros que lo deseaban, tenían la posibilidad de llevar a cabo varias reuniones para solventar cualquier duda o cuestión. A estas reuniones acudían generalmente el director/a y el orientador/a del centro, ya que serían los encargados de administrarlo posteriormente y devolverlo cumplimentado al Área de Orientación y Acceso del Vicerrectorado de Estudiantes de la Universidad de Oviedo. Tras llevar a cabo las aclaraciones pertinentes, cada centro educativo debía firmar el consentimiento informado para participar en el estudio; y una vez recibido el mismo, se les enviaba el número de copias necesarias del instrumento para llevar el estudio.

\section{ANÁLISIS DE LOS DATOS}

En primer lugar, se realizó un análisis descriptivo del contexto social y personal de los estudiantes. En segundo lugar, se calcularon los estadísticos univariados de los ítems (media, desviación típica, asimetría y curtosis) y la matriz de correlaciones entre los ítems.

Posteriormente, se estimó la dimensionalidad de la prueba. Para ello, la muestra se subdividió aleatoriamente en tres submuestras. En la primera $\left(n_{1}=656\right)$ se realizó un Análisis Factorial Exploratorio (AFE). La determinación del número de factores se realizó en función del método de Implementación Óptima de Análisis Paralelos propuesto por Timmerman y Lorenzo-Seva (2011) Ilevándose a cabo 5000 remuestreos. Se utilizó la correlación policórica como matriz de entrada de los datos, el método de factorización fue el de mínimos cuadrados no ponderados y el método de rotación Promin (Ferrando y Lorenzo-Seva, 2014). En la segunda submuestra $\left(n_{2}=697\right)$ se realizó un Análisis Factorial Confirmatorio (AFC) incluyendo las principales correlaciones entre los errores de medida de los ítems con la intención de obtener una representación más realista del constructo. Por último, la tercera submuestra $\left(n_{3}=652\right)$ se utilizó para realizar una validación cruzada del modelo previamente propuesto por lo que no se realizó ninguna modificación respecto al modelo anterior. El método de estimación de ambos modelos confirmatorios fue el de mínimos cuadrados ponderados robusto, indicado para datos ordenados categóricamente (Muthén y Muthén, 2012). La evaluación del ajuste de los datos al modelo se realizó en función de un criterio múltiple: $\chi 2 / g . l$, el Índice de Ajuste Comparativo (CFI), el Índice Tucker Lewis (TLI), la raíz cuadrática media de los residuales (RMSEA). La literatura sugiere que el ajuste puede considerarse adecuado cuando $\chi 2 / \mathrm{g}$.l. $<5, \mathrm{CFI}>.90, \mathrm{TLI}>.90$ y el RMSEA $<.08$ (Kline, $2011)$.

La estimación de la fiabilidad de las puntuaciones en las escalas se realizó mediante el Alfa para datos ordinales (Elosua y Zumbo, 2008) y el coeficiente de fiabilidad compuesta Omega de McDonald (McDonald, 1999). A continuación, se estimaron los parámetros y la función de información en el marco de la Teoría de Respuesta a los Ítems (TRI) utilizando el modelo de respuesta graduada (Samejima, 1969). Finalmente, se realizó la prueba de Kolmogorov-Smirnov para el estudio de la normalidad y las pruebas $U$ de Mann-Whitney y Kruskal-Wallis para estudiar las diferencias en el test de motivaciones en función del sexo y la titularidad del centro. La estimación del tamaño del efecto para pruebas no paramétricas se realizó mediante la probabilidad de superioridad (Grissom y Kim, 2011). Los datos fueron analizados utilizando el SPSS 19.0 (Arbuckle, 2010), el FACTOR 10 (Lorenzo-Sevay Ferrando, 2013), el MPLUS 7 (Muthén y Muthén, 19982012) y el FlexMIRT (Cai, 2013).

\section{RESULTADOS}

\section{CONTEXTO SOCIAL Y PERSONAL}

Con el fin de conocer en profundidad el tipo de estudiantes que cumplimentaron el cuestionario se analizaron las preguntas previas relacionadas con el contexto social y personal. En relación al contexto familiar, se comprobó que el $55.29 \%$ de los padres del alumnado participante no tenía estudios universitarios, el $22.46 \%$ poseía estudios universitarios (tanto el padre como la madre), el $15.23 \%$ solo la madre tenía estudios universitarios y el $9.21 \%$ 
solo el padre era el que poseía estudios de tipo universitario.

Asimismo, también se solicitó información sobre su futuro académico. A lo que el $80.30 \%$ respondió que pretendía acudir a la Universidad, mientras que un $11.10 \%$ no pensaba acudir a la Universidad, y el $8.6 \%$ restante aún no tenía claro que decisión tomar. Además, dentro del grupo de estudiantes que quería realizar algún grado universitario, solo el $63.60 \%$ de éstos tiene decidido qué carrera estudiar.

En este sentido, si relacionamos ambas variables, es decir, el tipo de estudios que poseen los padres con las perspectivas futuras del alumnado participante, se detectó que cuando el padre y la madre de los participantes habían estudiado en la Universidad, el $90.8 \%$ de los alumnos tenía perspectivas de ir a la Universidad, aunque solo un $62.8 \%$ de éstos ya tenían decidido qué carrera estudiar. Cuando solo la madre poseía estudios universitarios, el porcentaje de alumnos con perspectivas universitarias era del $86 \%$, de los que un $67.6 \%$ además tenía claro qué estudiar. Resultados similares se encontraron cuando era el padre el único con estudios universitarios, ya que el $85.70 \%$ del alumnado pretendía ir a la Universidad y solo $62 \%$ de ellos sabía qué estudios iba a llevar a cabo. Sin embargo, cuando ninguno de los padres posee estudios universitarios, el porcentaje de estudiantes con perspectivas futuras de acudir a la Universidad decrece a un $73.10 \%$, dentro de los cuáles el $62 \%$ tiene claro el tipo de grado en el que se matriculará y el $38 \%$ restante aún duda entre diferentes carreras o ni siquiera sabe qué estudiar.

Estos resultados han permitido comprobar que el nivel de estudios de los padres del alumnado

Tabla 1

Estadísticos descriptivos para cada uno de los ítems que conforman la escala motivaciones

\begin{tabular}{|c|c|c|c|c|c|}
\hline Ítems & $M$ & $D T$ & Asim & Curt. & $Z$ \\
\hline 1. Consejo y opinión de los padres & 4.93 & 2.95 & -0.25 & -0.93 & $5.75^{\star \star \star}$ \\
\hline 2. Proximidad de los estudios al domicilio familiar & 4.05 & 3.19 & 0.12 & -1.24 & $6.89 * * *$ \\
\hline 3. Opiniones de los amigos/as & 3.16 & 2.83 & 0.43 & -.919 & $7.86 * * *$ \\
\hline 4. Posibilidades que ofrece de encontrar trabajo & 6.72 & 2.65 & -1.02 & 0.42 & $8.06 * * *$ \\
\hline 5. Porque me gusta la carrera & 8.87 & 1.92 & -2.67 & 8.09 & $12.45^{\star \star \star}$ \\
\hline 6. Tengo aptitudes para esos estudios & 7.86 & 1.97 & -1.56 & 3.34 & $9.20 * \star \star$ \\
\hline 7. Es lo que más me gusta y siempre lo he querido estudiar & 7.40 & 2.66 & -1.15 & 0.73 & $7.81^{* * *}$ \\
\hline 8. Prestigio de la carrera & 5.12 & 2.98 & -0.40 & -0.89 & $6.69 * * *$ \\
\hline 9. Nota de corte para entrar & 4.79 & 3.35 & -0.115 & -1.21 & $6.21 * * *$ \\
\hline 10. Consejos de mis profesores/as y/o tutores/as & 3.99 & 3.10 & 0.05 & -1.26 & $7.29 * * *$ \\
\hline $\begin{array}{l}\text { 11. Asesoramiento del/a Orientador/a (psicólogo/a, pedagogo/a, } \\
\text { psicopedagogo/a de mi centro) }\end{array}$ & 2.37 & 2.95 & 0.88 & -0.578 & $13.47^{* * *}$ \\
\hline 12. Que es una carrera fácil & 2.38 & 2.72 & 0.82 & -0.428 & $11.70 * * *$ \\
\hline 13. Prestigio de la Universidad & 3.89 & 3.18 & 0.12 & -1.27 & $8.09 * * *$ \\
\hline $\begin{array}{l}\text { 14. Tradición familiar (es la profesión de mi padre/madre o un } \\
\text { familiar cercano) }\end{array}$ & 1.31 & 2.50 & 1.88 & 2.44 & $18.56 * \star *$ \\
\hline 15. Deseo de saber más sobre ese campo & 7.13 & 2.82 & -1.14 & 0.57 & $8.09 * * *$ \\
\hline 16. Un/a profesional de mi entorno con esos estudios & 3.23 & 3.45 & 0.543 & -1.18 & $11.75^{\star \star \star}$ \\
\hline
\end{tabular}

Nota: $M=$ Media, DT= Desviación Típica, Asim.= Asimetría, Curt.= Curtosis, Z= Z de Kolmogorov Smirnov. Puntuación Mínima Teórica $=0$; Puntuación Máxima Teórica $=10$.

${ }^{*} p<.05 ;{ }^{*} p<.01 ; * * * p<.001$. 
participante modifica ligeramente la tendencia de acudir o no a la Universidad, aunque no interfiere en el grado de decisión sobre la carrera a elegir.

\section{ANÁLISIS DE LAS MOTIVACIONES PARA LA ELECCIÓN DE CARRERA UNIVERSITARIA}

En la Tabla 1 se muestran los estadísticos descriptivos calculados para cada motivo. Se ha detectado que el porcentaje de estudiantes que no han contestado a alguno de los ítems es menor al $2 \%$, es decir, prácticamente la totalidad de los participantes han cumplimentado al completo la escala, lo que sugiere que los diferentes ítems son claros y no llevan a confusión.

Cuando se analizaron los índices de asimetría y curtosis, así como la Z de Kolmogorov-Smirnov, se comprobó que las puntuaciones procedentes de la muestra participante no siguen una distribución normal. Esto es debido a que si bien en algunos ítems se detecta una asimetría positiva (ítems: 2, $3,10,11,12,13,14$ y 16) en otros ítems hay una asimetría negativa (ítems: 1, 4, 5, 6, 7, 8, 9 y 15).

Por otro lado, se analizaron los estadísticos descriptivos de la escala para detectar qué tipo de motivaciones tienen más importancia en la elección de estudios universitarios. Los resultados mostraron que el ítem 5 es él que ha recibido una mayor puntuación, seguido de otros ítems (como el ítem 4, 6, 7, 8 y 15) que han obtenido puntuaciones igualmente elevadas. Es decir, las motivaciones intrínsecas relacionadas con aspectos vocacionales e intereses personales ("quiero saber más sobre ese campo", "porque me gusta la carrera"...) han sido las más valoradas. Por su parte, dentro de las motivaciones extrínsecas, los

Tabla 2

Correlaciones inter-ítem de la escala

\begin{tabular}{|c|c|c|c|c|c|c|c|c|c|c|c|c|c|c|c|c|}
\hline It & 1 & 2 & 3 & 4 & 5 & 6 & 7 & 8 & 9 & 10 & 11 & 12 & 13 & 14 & 15 & 16 \\
\hline 1 & 1 & & & & & & & & & & & & & & & \\
\hline 2 & $.317^{* *}$ & 1 & & & & & & & & & & & & & & \\
\hline 3 & $.317^{* *}$ & $.282^{* *}$ & 1 & & & & & & & & & & & & & \\
\hline 4 & $.344^{* *}$ & $.247^{* *}$ & $.254^{* *}$ & 1 & & & & & & & & & & & & \\
\hline 5 & $-.067^{* *}$ & -.031 & $-.061^{* *}$ & $.053^{* *}$ & 1 & & & & & & & & & & & \\
\hline 6 & -.023 & .004 & -.020 & $.101^{* *}$ & $.489^{* *}$ & 1 & & & & & & & & & & \\
\hline 7 & $-.057^{*}$ & -.020 & -.033 & -.002 & $.478^{* *}$ & $.430^{* *}$ & 1 & & & & & & & & & \\
\hline 8 & $.255^{* *}$ & $.173^{* *}$ & $.238^{* *}$ & $.408^{* *}$ & $.101^{* *}$ & $.167^{* *}$ & 年 & 1 & & & & & & & & \\
\hline 9 & $.166^{* *}$ & $.235^{* *}$ & $.194^{* *}$ & $.217^{* *}$ & -.015 & -.023 & -.015 & $.268^{* *}$ & 1 & & & & & & & \\
\hline 10 & $.396^{* *}$ & $.278^{* *}$ & $.387^{* *}$ & $.268^{* *}$ & -.005 & $.055^{*}$ & .033 & $.293^{* *}$ & $.276^{* *}$ & 1 & & & & & & \\
\hline 11 & $.253^{* *}$ & $.236^{* *}$ & $.273^{* *}$ & $.164^{* *}$ & $-.064^{* *}$ & -.024 & .031 & $.187^{* *}$ & $.212^{* *}$ & $.519^{* *}$ & 1 & & & & & \\
\hline 12 & $.179^{* *}$ & $.187^{* *}$ & $.287^{* *}$ & $.173^{* *}$ & $-.136^{\star *}$ & $-.080^{* *}$ & $-.065^{* *}$ & $.170^{* *}$ & $.374^{* *}$ & $.245^{* *}$ & $.326^{* *}$ & 1 & & & & \\
\hline 13 & $.274^{* *}$ & $.180^{* *}$ & $.250^{* *}$ & $.256^{* *}$ & $.055^{*}$ & $.113^{* *}$ & $.085^{* *}$ & $.487^{* *}$ & $.252^{* *}$ & $.290^{* *}$ & $.268^{* *}$ & $.297^{* *}$ & 1 & & & \\
\hline 14 & $.226^{* *}$ & $.137^{* *}$ & $.169^{* *}$ & $.106^{* *}$ & $-.096^{* *}$ & -.016 & .022 & $.165^{* *}$ & .117** & $.145^{* *}$ & $.187^{* *}$ & $.219^{* *}$ & $.222^{* *}$ & 1 & & \\
\hline 15 & -.020 & -.023 & -.006 & -.018 & $.332^{* *}$ & $.266^{* *}$ & $.276^{* *}$ & $.103^{* *}$ & -.027 & .028 & -.040 & $-.131^{* *}$ & $.129^{* *}$ & .010 & 1 & \\
\hline 16 & $.199^{* *}$ & $.128^{* *}$ & $.173^{* *}$ & $.156^{* *}$ & -.015 & $.056^{*}$ & $.068^{* *}$ & $.209^{* *}$ & $.165^{* *}$ & $.206^{* *}$ & $.177^{* *}$ & $.207^{* *}$ & $.217^{* *}$ & $.383^{* *}$ & $.133^{* *}$ & 1 \\
\hline
\end{tabular}


ítems referidos al prestigio de la Universidad o las futuras salidas laborales, han sido las que han recibido una mayor puntuación, aunque significativamente menor si las comparamos con los motivos de origen intrínseco.

Por lo que se refiere a las correlaciones de Pearson entre ítems (Tabla 2), se ha observado que la mayor correlación es de .519.A la vista de la matriz de correlaciones, se observa que no se trata de una estructura unidimensional ya que existen ítems con bajas correlaciones entre ellos, por ejemplo, los ítems 5, 6, 7 y 15.

\section{DIMENSIONALIDAD DE LA PRUEBA}

En la primera submuestra se realizó un Análisis Factorial Exploratorio. El índice Kaiser Meyer Olkin (KMO > .70) y la prueba de Barlett $(p<.001)$ mostraron que la matriz de datos es adecuada para su factorización. En función del método de Implementación Óptima de Análisis Paralelos se extraen dos factores. El primer factor, denominado motivaciones intrínsecas, explica el $25.69 \%$ de la varianza y el segundo factor, motivaciones extrínsecas, el 15.70\%. Los índices de ajuste mostraron un ajuste de los datos al modelo $(G F I=.97 ;$ RMSR $=.066)$. Los resultados del AFC realizado en la segunda submuestra permiten confirmar el ajuste de los

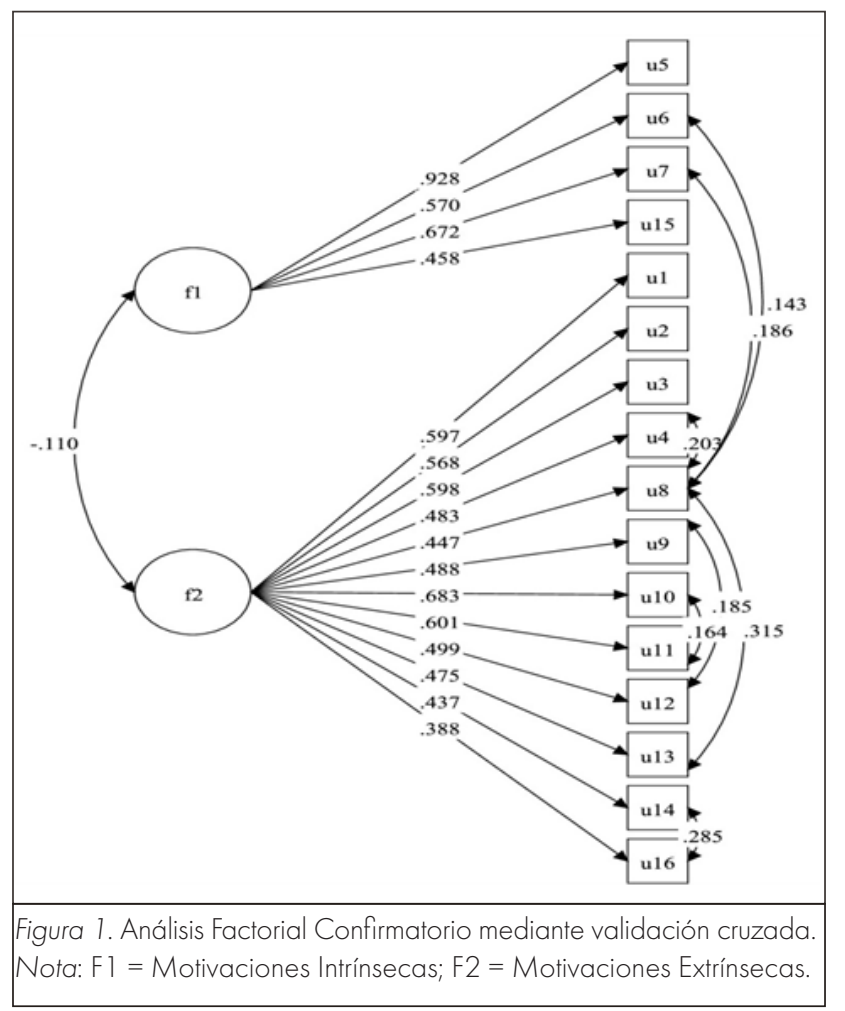

datos al modelo $(\chi 2 / \mathrm{gl}=376.61 / 96=3.92 ;$ $\mathrm{CFI}=.92 ; \mathrm{TLI}=.90 ; \mathrm{RMSEA}=.066[\mathrm{IC} 90 \%$ $=.059-.0773])$. En la tercera submuestra se realizó la validación cruzada del modelo, la cual también mostró un ajuste adecuado $(\chi 2 / \mathrm{gl}$ $=319.73 / 96=3.33 ; \mathrm{CFI}=.94 ; \mathrm{TLI}=.92$; RMSEA $=.060$ [IC 90\% $=.053-.068])$. Como se puede observar en la figura 1, todos los pesos fueron superiores a .30 y la correlación entre factores fue de -. 11 .

\section{FIABILIDAD DE LAS PUNTUACIONES}

El coeficiente Alfa para datos ordinales correspondiente a la primera subescala fue .82 y el Omega de McDonald .77. Para la segunda subescala el Alfa ordinal fue de .83 y el Omega de McDonald .82. Los índices de discriminación para la primera subescala oscilaron entre .37 y .58 mientras que para la segunda subescala oscilaron entre .32 y .54 .

\section{PARÁMETROS DE LA TEORÍA DE RESPUESTA A LOS ÍTEMS}

Los parámetros de discriminación de la primera subescala oscilaron entre 1.05 y 2.86 y los de la segunda subescala entre .83 y 1.71 . Sin bien los primeros pueden considerarse ítems con un alto poder discriminativo ambos conjuntos de ítems tienen un poder discriminativo adecuado (Baker, 2001). En las figuras 2 y 3 se presentan las funciones de información de ambas subescalas. La primera proporciona los

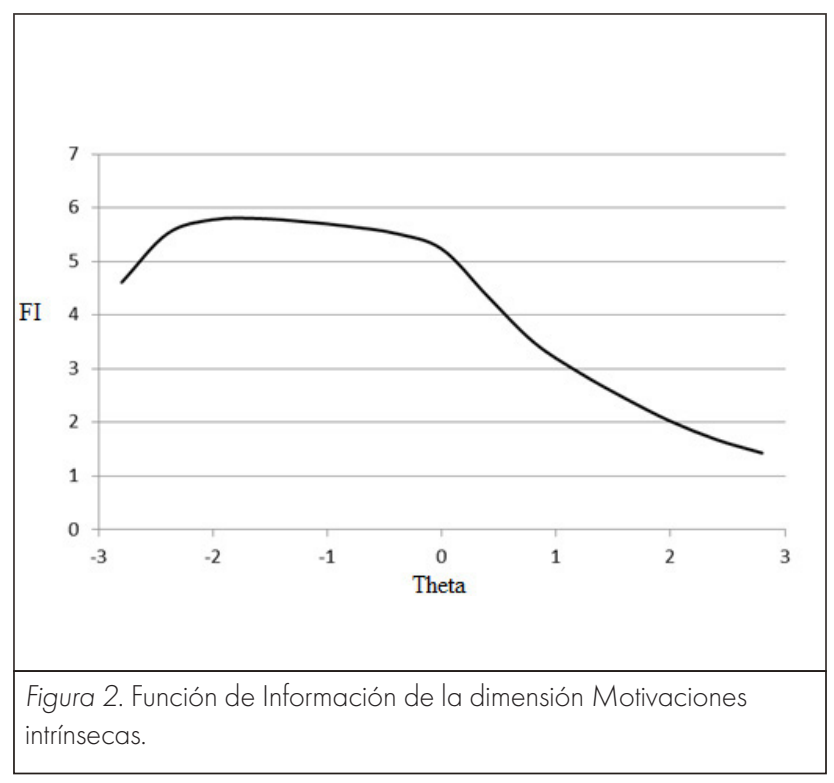




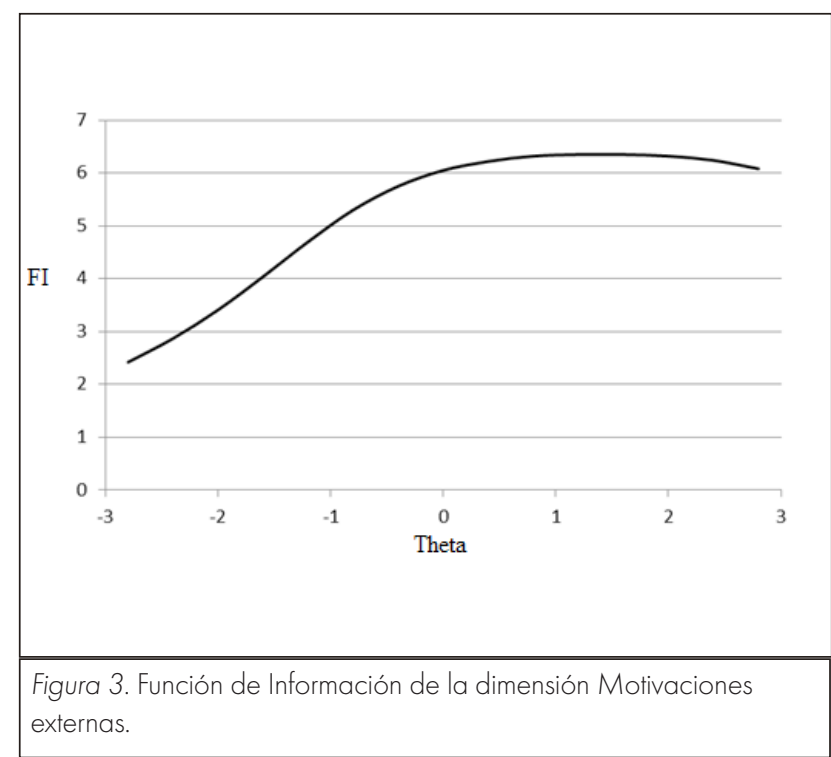

mayores niveles de precisión para puntuaciones theta entre -2 y 0 . En cambio, la segunda proporciona mayores niveles de precisión para puntuaciones theta entre $0 y+3$.

\section{DIFERENCIAS EN MOTIVACIONES EN FUNCIÓN DEL SEXO Y LA TITULARIDAD DEL CENTRO EDUCATIVO}

La prueba de Kolmogorov-Smirnov mostró que la distribución de puntuaciones no se ajusta a una distribución normal en ninguna de las dos subescalas ( $p<$.05). Además, el estadístico de asimetría fue de - $1.28($ E.T. $=.055)$ y el de curtosis de $2.16($ E.T. $=.11$ ), lo que indica un mal ajuste a la distribución normal. A continuación, se realizó la prueba $U$ de Mann-Whitney para el estudio de las diferencias en motivación en función del sexo.

En la Tabla 3, se muestra que existen diferencias estadísticamente significativas en función del sexo en la primera subescala a favor de las mujeres. Además, el tamaño del efecto muestra que las diferencias podrían considerarse moderadas. Para estudiar las diferencias en función de la titularidad del centro se realizó la prueba de Kruskal-Wallis. Los resultados muestran que existen diferencias estadísticamente significativas en función de la titularidad en ambas subescalas. En concreto, las comparaciones múltiples a posteriori muestran que estas diferencias se encuentran principalmente entre los centros públicos y privados, obteniendo estos últimos mayores puntuaciones en ambas subescalas. Además, el tamaño del efecto muestra que estas diferencias podrían considerarse moderadas.

\section{DISCUSIÓN}

Dado que el primero de los objetivos trataba de analizar las propiedades psicométricas del cuestionario, los resultados respaldan que éste muestra unas propiedades psicométricas adecuadas. La totalidad de los ítems contribuye a medir la variable motivaciones en la elección de estudios universitarios en una misma dirección. En cuanto a su estructura factorial, los resultados han demostrado que las diferentes motivaciones convergen en dos factores claramente diferenciados. En este sentido, considerandolas aportaciones procedentes de investigaciones previas (Lerchundi, Núñez del Río,y GonzálezTirados, 2015; Savage, Bich, y Noussi, 2011), ambos factores podrían clasificarse en dos grandes grupos de motivaciones: motivaciones intrínsecas y motivaciones extrínsecas. De este modo, tal y como describían Ryan y Deci (2000), las motivaciones intrínsecas son las que dependen del estudiante, como los gustos y

Tabla 3

Diferencias en el test de motivaciones en función del sexo y la titularidad del centro

\begin{tabular}{|c|c|c|c|c|c|c|c|c|c|c|c|}
\hline \multicolumn{6}{|c|}{ Sexo } & \multicolumn{6}{|c|}{ Titularidad } \\
\hline & $\mathrm{R}(\mathrm{H})$ & $\mathrm{R}(\mathrm{M})$ & z & $\mathrm{p}$ & T.E. & $\mathrm{R}(\mathrm{Pb})$ & $\mathrm{R}$ (Con) & $\mathrm{R}$ (Priv) & $\chi^{2(g . l .)}$ & $\mathrm{p}$ & Post-hoc \\
\hline $\mathrm{F} 1$ & 928.83 & 1017.49 & -3.47 & .001 & .45 & 959.20 & 1004.81 & 1090.52 & $10.84(2)$ & .004 & $\begin{array}{l}\text { Priv> } \\
\mathrm{Pb}^{*} 1\end{array}$ \\
\hline $\mathrm{F} 2$ & 994.13 & 956.11 & -1.49 & .137 & .48 & 954.14 & 993.26 & 1106.87 & $14.13(2)$ & .001 & $\begin{array}{l}\text { Priv }> \\
\mathrm{Pb} \approx 2\end{array}$ \\
\hline
\end{tabular}

Nota. F1 = Motivos intrínsecos; F2 = Motivos extrínsecos; $R(H)=$ Rango promedio hombres; $R(M)=$ Rango promedio mujeres; $R(P b)=$ Rango promedio centros públicos; R(Con) = Rango promedio centros concertados; R(Priv) = Rango promedio centros privados; T.E. = Estimación del tamaño del efecto mediante la Probabilidad de Superioridad * $p<.001$; 1T.E. = .43; 2 T.E. $=.42$. 
las preferencias personales así como aquellos aspectos relacionados con la vocación hacia una determinada disciplina. En cambio, las motivaciones extrínsecas hacen referencia a aspectos que no dependen del propio individuo sino de factores o circunstancias externascomo el prestigio de la Universidad, las salidas laborales, o los consejos recibidos por parte de los padres y profesores.

En cuanto al objetivo central del estudio, de acuerdo a lo planteaban distintos estudios de carácter nacional e internacional (Bowdeny Wood, 2011; Lubben et al., 2010; Skatova y Ferguson, 2014), las preferencias y los intereses personales son los motivos más influyentes a la hora de elegir qué carrera estudiar. Es decir, los estudiantes eligen según lo que les gusta e interesa, y pasan a un segundo puesto otros aspectos que pueden ser fundamentales en su futuro como las salidas laborales que ofrecen los diferentes grados universitarios. En este sentido, se podría afirmar que las motivaciones intrínsecas tienen más peso en la elección de estudios universitarios que aquellas de tipo extrínseco.

Por otro lado, la tradición familiar, la facilidad de la carrera o el asesoramiento del orientador del centro, han sido las motivaciones extrínsecas que menos influyen en la elección de un determinado grado universitario. Estos resultados han puesto de manifiesto la necesidad de mejorar los programas de orientación vocacional de los centros educativos con el objetivo de recibir una mejor valoración por parte del alumnado de Bachillerato. Una posible explicación de estos datos, podría ser que los estudiantes de Bachillerato reciben menor atención por parte del departamento de orientación puesto que la hora de tutoría que tenían en la ESO desaparece durante esta nueva etapa y solo tienen atención del departamento de orientación bajo demanda de los padres o el tutor/a.

Además, si se atiende a la diferenciación según el género, los resultados mostraron que en las mujeres tienen mayor peso que en los hombres las motivaciones intrínsecas, en espacial aquellas que hacen referencia a aspectos de tipo vocacional ("es la carrera que siempre me ha gustado", "quiero saber más sobre ese campo", "tengo aptitudes y habilidades para estos estudios"). Este hallazgo podría explicar el hecho de que las mujeres obtengan mejores calificaciones que los hombres en carreras universitarias (Rodríguez, Fita, y Torrado, 2004), puesto que varias investigaciones han señalado que cuando el alumnado estudia con una motivación intrínseca, éste tiene más probabilidades de encontrar el éxito académico siempre que ésta se acompañe de un estilo de aprendizaje profundo (CasoNiebla y Hernández, 2007; Cerezo, Núñez, Fernández, Suárez-Fernández y Tuero, 2011; Colmenares y Delgado, 2010).

Asimismo, otra variable que se ha tenido en cuenta en la presente investigación, hace referencia al tipo de centro en el que se encuentra el alumnado participante. Esta variable ha mostrado ser relevante, ya que se ha comprobado como todos aquellos motivos de tipo extrínseco que tienen que ver con el centro ("Consejos de mis profesores/tutores", "asesoramiento del orientador") son mejor valorados por los estudiantes de los centros concertados y privados que por los estudiantes de los centros públicos. En este sentido, parece que las actividades de orientación del sector privado adquieren una mayor relevancia que las del sector público.

Tales resultados han evidenciado la fiabilidad y eficacia del nuevo cuestionario para analizar las motivaciones más relevantes en el alumnado de segundo de Bachillerato. La utilidad de este cuestionario no solo se reduce a conocer los motivos por los que el alumnado elige un estudio u otro, sino que también sirve para vislumbrar aspectos a mejorar de los centros educativos, así como para conocer el contexto familiar del alumno (tipos de estudios del padre o madre) y las perspectivas futuras de ir o no a la Universidad. Todos estos datos pueden resultar especialmente relevantes para el centro educativo, ya que permiten al profesorado conocer las opiniones del alumnado en mayor profundidad. En este sentido, dado que el motivo principal para elegir estudios universitarios se basa en sus interese personales, resultaría 
beneficioso aportarles información sobre las carreras de interés (preferentemente utilizando las páginas de las facultades), especificando el tipo de competencias a desempeñar, lo que les facilitará la toma de decisiones.

Por último, es importante señalar aquellas limitaciones que deben ser solventadas en líneas futuras. Estas limitaciones están relacionadas con el tipo de muestra utilizada, ya que sería conveniente que el cuestionario fuera aplicado a otras comunidades con el fin de observar si los motivos mejor valorados coinciden con los analizados en la presente investigación. Además, sería recomendable aumentar las evidencias de validez del instrumento desarrollado (Lane, 2014; Padilla y Benítez, 2014; Ríos y Wells, 2014; Sireci y Faulkner-Bond, 2014). Por su parte, otra limitación del estudio se basa las limitaciones inherentes al uso de autoinformes. Es posible que, en ocasiones, el informante no sea sincero, lo que impide conocer con certeza si el alumnado ha elegido la carrera que afirma en el autoinforme, así como comprobar si las motivaciones señaladas en el mismo son las motivaciones reales de la elección.

En cuanto a las líneas futuras de investigación, del mismo modo que se ha analizado la escala de motivos, sería conveniente analizar la escala relacionada con el uso y utilidad de las diferentes fuentes de información con el propósito de conocer aquellas fuentes mejor valoradas y más utilizadas por los estudiantes de Bachillerato. Asimismo, con objeto de extender los resultados a otras comunidades autónomas, actualmente se está desarrollando una herramienta informática para cumplimentar la encuesta online y descargar la información automáticamente permitiendo su aplicación a nivel nacional.

\section{- Conflicto de intereses}

Los autores declaran no tener ningún conflicto de intereses.

\section{REFERENCIAS}

Álvarez, P.R., Cabrera, L., González, M. C., y Bethencourt, J. T. (2006). Causas del abandono y prolongación de los estudios universitarios. Paradigma, 27(1), 349-363.

American Educational Research Association, American Psychological Association, and National Council on Measurement in Education. (2014). Standards for educational and psychological testing. Washington, DC: American Psychological Association.

Arbuckle, J. L. (2010). SPSS (Statistical Package for the Social Sciences) (Version 19.0) [Computer program]. Chicago, IL: SPSS.

Areces, D., Rodríguez-Muñiz, L.J., SuárezÁlvarez, J., De la Roca, Y., y Cueli, M. (2016). Fuentes de información que utilizan los estudiantes de bachillerato en la elección de carrera universitaria. Psicothema, 28(3), 253259. doi: 10.7334/psicothema2016.76

Baker, F. (2001). The basics of item response theory. University of Maryland: College Park: ERIC Clearinghouse on Assessment and Evaluation

Bowden, J., y Wood, L. (2011). Sex doesn'† matter: The role of gender in the formation of student-university relationships. Journal of Marketing for Higher Education, 21 (2), 133156. doi:10.1080/08841241.2011.623731

Cai, L. (2013). flexMIRT version 2: Flexible multilevel multidimensional item analysis and test scoring [Computer software]. Chapel Hill, NC: Vector Psychometric Group.

Canales, A., y De los Ríos, D. (2018). Factores explicativos de la deserción universitaria. Calidad en la Educación, 26.

Candela, C. (2008). Motivaciones y expectativas profesionales. Análisis desde la perspectiva de género (Tesis doctoral, Universidad de Valencia). Recuperada de: http://roderic. uv.es/handle/10550/15419

Caso-Niebla, J., y Hernández, L. (2007). Variables que inciden en el rendimiento académico de adolescentes mexicanos. Revista Latinoamericana de Psicología, 39(3), 487-501.

Cerezo, R., Núñez, J. C., Fernández, E., SuárezFernández, N., y Tuero, E. (2011). Programas de intervención para la mejora de las competencias de aprendizaje autorregulado en educación superior. Perspectiva Educacional, 50(1), 1-30. 
Colmenares, M., y Delgado, F. (2010). La correlación entre rendimiento académico y motivación de logro: elementos para la discusión y reflexión. Redhecs, 5(3), 179 191.doi:10.1037/0022-3514.37.9.1487

Conroy,D.E.,Elliot,A.J.,y Pincus, A. L.(2009). The expression of achievement motives in interpersonal problems. Journal of Personality, 77(2), 495-526. doi:10.1111/i.14676494.2008.00556.x.

Deemer, E. D., Martens, M. P., y Buboltz, W. C. (2010). Toward a tripartite model of research motivation: development and initial validation of the research motivation scale. Journal of Career Assessment, 18(3), 292309. doi:10.1177/1069072710364794

De la Mella Quintero, S. F., Díaz, J. M. M., y Pérez, J. M. C. (2013). Factores motivacionales que influyeron en estudiantes de primer año para elegir la carrera de Estomatología. Edumecentro, 4(3), 188-197.

Elosua, P., y Zumbo, B. D. (2008). Reliability coefficients for ordinal response scales. Psicothema, 20(4), 896-901.

Ferrando, P.J. y Lorenzo-Seva, U. (2014). El análisis factorial exploratorio de los ítems: Algunas consideraciones adicionales. Anales de Psicología 30(3), 1170-1175.

Gámez, E., y Marrero, H. (2000). Metas y motivos en la elección de la carrera de Psicología. Revista Electrónica de Motivación y Emoción, 3, 5-6.

Gámez, E., y Marrero, H. (2003). Metas y motivos en la elección de la carrera universitaria: Un estudio comparativo entre psicología, derecho y biología. Anales de Psicología, 19 (1), 24-36. doi:10.6018/ analesps.31.2.171851

Grissom, R.J., y Kim, J.J. (2011). Effect Sizes for Research: Univariate and Multivariate Applications, Second Edition. New York, NY: Routledge.

Haladyna, T.M., y Rodriguez, M.C. (2013). Developing and validating test ítems. New York, NY: Routledge.

Kline, R. B. (2011). Principles and practice of structural equation modeling. New York: Guilford Press.
Lane, S. (2014). Validity evidence based on testing consequences. Psicothema, 26, 127 135. doi: 10.7334/psicothema2013.258

Lerchundi, P., Núñez-del-Río, M. C., y GonzálezTirados, R. M. (2015). Career choice in engineering students: its relationship with motivation, satisfaction and the development of professional plans. Anales de Psicología, 31 (1), 268-279. doi:10.6018/ analesps.31.1.159161

López, M. D., González, A., y Rodríguez, L. M. (2009). Motivaciones de Carrera, Creencias irracionales y Competencia personal en Estudiantes de Psicología. Revista de Enseñanza de la Psicología: Teoría y Experiencia, 5(1), 50-63.

Lorenzo, M., Argos, J., Hernández, J., y Vera, J. (2014). El acceso y la entrada del estudiante a la universidad: Situación y propuestas de mejora facilitadoras del tránsito. Educación XX1, 17 (1), 15-38. doi:10.5944/ educxx1.17.1.9951.

Lorenzo-Seva, U. y Ferrando, P. J. (2013). Manual of the program FACTOR v. 9.20. Recuperado de http://psico.fcep.urv.es/utilitats/factor/ documentation/Manual-of-the-FactorProgram-v92.pdf

Lubben, F., Davidowitz, B., Buffler, A., Allie, S., y Scott, I. (2010). Factors influencing access students' persistence in an undergraduate science programme: A South African case study. International Journal of Educational Development, 30(4), 351-358. doi: 10.1016/i.ijedudev.2009.11.009

McDonald, R.P. (1999). Test theory: A unified treatment. Mahwah, NJ: Lawrence Erlbaum.

Martínez, A., Zurita, F., Castro, M., Chacón, R., Hinojo, M. A., y Espejo, T. (2016). La elección de estudio superiores universitarios en estudiantes de último curso de bachillerato y ciclos formativos. Revista Electrónica Educare, 20(1), 1-18. doi: 10.15359/ree.20-1.14.

Mendoza-León, T., y Rodríguez, R. (2008). El efecto de la orientación vocacional en la elección de carrera. Revista Mexicana de Orientación Educativa, 5(13), 10-16.

Moreno, R., Martínez, R., y Muñiz, J. (2015). Guidelines based on validity criteria for 
the development of multiple choice items. Psicothema, 27(4), 388-394. doi: 10.7334/ psicothema2015.110

Muthén, L.K. and Muthén, B.O. (1998-2012). Mplus User's Guide. Seventh Edition. Los Angeles, CA: Muthén y Muthén.

Padilla, J.L., y Benítez, I. (2014). Validity evidence based on response processes. Psicothema, 26, 136-144. doi: 10.7334/psicothema2013.259

Pérez Morán, J. C., Talavera Chávez, R., y Ramos Vargas, A. A. (noviembre, 2013). Análisis del abandono, del proceso de elección y del cambio de carrera en estudiantes universitarios. Trabajo presentado en la Conferencia Latinoamericana sobre el Abandono en la Educación Superior, México, DF. Resumen recuperado de http://www.alfaguia.org/ www-alfa/images/ponencias/clabesIII/LT_1/ ponencia completa 108.pdf

Peró, M., Soriano, P. P., Capilla, R., Olmos, J. G., y Hervás, A. (2015). Questionnaire for the assessment of factors related to university degree choice in Spanish public system: A psychometric study. Computers in Human Behavior, 47, 128-138. doi: 10.1016/i. chb.2014.09.003

Rios, J., y Wells, C. (2014). Validity evidence based on internal structure. Psicothema, 26, 108116. doi: 10.7334/psicothema2013.260

Rodríguez, S., Fita, E., y Torrado, M. (2004). El rendimiento académico en la transición secundaria-universidad. Revista de Educación, $334,391-414$.
Ryan, R.M., y Deci, E.L. (2000). Intrinsic and Estrinsic motivations: classic definitions and new directions. Contemporary Educational Psychology, 25, 54-69.

Samejima, F. (1969). Estimation of latent ability using a response pattern of graded scores. Psychometrika Monograph No. 17.

Savage, N., Birch, R., y Noussi, E. (2011). Motivation of engineering students in higher education. Engineering Education, 6(2), 3946. doi:10.11120/ened.2011.06020039

Sireci, S., y Faulkner-Bond, M. (2014). Validity evidence based on test content. Psicothema, 26, 100-107. doi: 10.7334/psicothema2013.256

Skatova, A., y Ferguson, E. (2014). Why do different people choose different university degrees? Motivation and the choice of degree. Frontiers in Psychology, 5, 1-15. doi:10.3389/ fpsyg.2014.01244

Soria, M., Guerra, M., Giménez, I., y Escanero, J. F. (2006). La decisión de estudiar medicina: características. Educación Médica, 9(2), 91-97. doi:10.4321/S1575-18132006000200008

Timmerman, M. E., y Lorenzo-Seva, U. (2011). Dimensionality assessment of ordered polytomous items with parallel analysis. Psychological Methods, 16, 209-220. doi:10.1037/a0023353 


\section{ANEXO \\ CUESTIONARIO SOBRE LA ELECCIÓN DE ESTUDIOS UNIVERSITARIOS}

Desde la Red de Servicios de Información y Orientación Universitarios (SIOU) deseamos conocer tu opinión sobre varias cuestiones relacionadas con la elección de estudios para replantear las actividades que organizamos para vosotros/as. Tus respuestas son muy importantes, contesta con total sinceridad, los datos son estrictamente confidenciales. Agradecemos sinceramente tu colaboración.

Año de nacimiento (dd/mm/aaaa):

Sexo: Hombre

Mujer

Provincia en la que estudias:

Tu Especialidad en el Bachillerato:

Humanidades
Ciencias y Tecnología
Ciencias Sociales
Artes

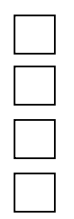

Centro en el que estudias:

Público $\square \quad$ Concertado

Privado $\square$

¿Tienes alguna discapacidad?:

Sí $\square$ No

¿Cuál?

¿̇Tiene tu padre estudios universitarios?:

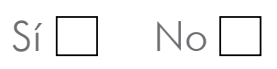

¿̇Tiene tu madre estudios universitarios?:

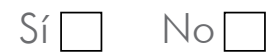

¿Piensas ir a la Universidad?

Sí $\square$ ¿̇Has decidido qué estudios universitarios quieres comenzar el próximo curso? ¿Qué Grado has decidido cursar?

No $\square$ ¿Qué tienes pensado hacer el próximo curso?

Dudoso

1. A continuación, te planteamos algunas cuestiones sobre los motivos que te han llevado a elegir la carrera universitaria. Tienes que responder en una escala de 1 a 10, dónde el 1 significa muy poca influencia y el 10 es la máxima. Utiliza los números intermedios de forma razonable. En el caso de que no pienses ir a la Universidad, o aún no hayas decidido la carrera que vas a estudiar, nos interesa igualmente tu opinión sobre la importancia que das a estas cuestiones. 
¿Qué motivos consideras que han influido o podrían influir en tu elección de la carrera?

\begin{tabular}{|c|c|c|c|c|c|c|c|c|c|c|}
\hline Consejo y opinión de los padres & 1 & 2 & 3 & 4 & 5 & 6 & 7 & 8 & 9 & 10 \\
\hline $\begin{array}{l}\text { Proximidad de los estudios al domicilio } \\
\text { familiar }\end{array}$ & 1 & 2 & 3 & 4 & 5 & 6 & 7 & 8 & 9 & 10 \\
\hline Opiniones de los amigos/as & 1 & 2 & 3 & 4 & 5 & 6 & 7 & 8 & 9 & 10 \\
\hline Posibilidades que ofrece de encontrar trabajo & 1 & 2 & 3 & 4 & 5 & 6 & 7 & 8 & 9 & 10 \\
\hline Porque me gusta la carrera & 1 & 2 & 3 & 4 & 5 & 6 & 7 & 8 & 9 & 10 \\
\hline Tengo aptitudes para esos estudios & 1 & 2 & 3 & 4 & 5 & 6 & 7 & 8 & 9 & 10 \\
\hline $\begin{array}{l}\text { Es lo que más me gusta y siempre lo he } \\
\text { querido estudiar }\end{array}$ & 1 & 2 & 3 & 4 & 5 & 6 & 7 & 8 & 9 & 10 \\
\hline Prestigio de la carrera & 1 & 2 & 3 & 4 & 5 & 6 & 7 & 8 & 9 & 10 \\
\hline Nota de corte para entrar & 1 & 2 & 3 & 4 & 5 & 6 & 7 & 8 & 9 & 10 \\
\hline Consejos de mis profesores/as y/o tutores/as & 1 & 2 & 3 & 4 & 5 & 6 & 7 & 8 & 9 & 10 \\
\hline $\begin{array}{l}\text { Asesoramiento del/a Orientador/a } \\
\text { (psicólogo/a, pedagogo/a, psicopedagogo/a } \\
\text { de mi centro) }\end{array}$ & 1 & 2 & 3 & 4 & 5 & 6 & 7 & 8 & 9 & 10 \\
\hline Que es una carrera fácil & 1 & 2 & 3 & 4 & 5 & 6 & 7 & 8 & 9 & 10 \\
\hline Prestigio de la Universidad & 1 & 2 & 3 & 4 & 5 & 6 & 7 & 8 & 9 & 10 \\
\hline $\begin{array}{l}\text { Tradición familiar (es la profesión de mi } \\
\text { padre/madre o un familiar cercano) }\end{array}$ & 1 & 2 & 3 & 4 & 5 & 6 & 7 & 8 & 9 & 10 \\
\hline Deseo de saber más sobre ese campo & 1 & 2 & 3 & 4 & 5 & 6 & 7 & 8 & 9 & 10 \\
\hline $\begin{array}{l}\text { Un/a profesional de mi entorno con esos } \\
\text { estudios }\end{array}$ & 1 & 2 & 3 & 4 & 5 & 6 & 7 & 8 & 9 & 10 \\
\hline
\end{tabular}

Otras razones, señala cuáles: 